\title{
Propiedades psicométricas del cuestionario para medir estrategias de aumento de la felicidad (HIS) en versión castellana (CEA-EAP).
}

\section{Psychometric properties of the questionnaire to measure strategies to increase happiness (HIS) in Spanish version (CEA-EAP).}

\author{
Emilio Moyano-Díaz \\ Universidad de Talca, Chile \\ Darío Páez \\ Universidad del País Vasco, España \\ Mauricio Torres \\ Universidad de Talca, Chile
}

Rec (07 agosto 2015) Acept (25 junio 2016)

\begin{abstract}
Resumen
Se adaptó al contexto chileno la Escala de Incremento de la Felicidad (HIS, de Tkach y Lyubomirsky, 2006) para medir estrategias conductuales de incremento de la felicidad, con el nombre de Cuestionario de Estrategias para Aumentar los Estados de Ánimo Positivos (CEA-EAP) con 44 ítems en formato Likert de 4 puntos. Este, junto con la EFS (Lyubomirsky y Lepper 1999) fue respondido por 443 estudiantes universitarios (Cronbach global de .80), quienes se autoclasificaron como extra o introvertidos. El ANAFAC mostró una estructura diferente a la original de ocho factores y mayoritariamente con buena confiabilidad. Las estrategias de control mental y auto-cuidado o reevaluación positiva y reorientación positiva de la atención, las de sociabilización recreativa o festejar y, en menor medida pero también otras tres diferentes estrategias, se asociaron a felicidad (EFS), y a la autoclasificación de extrovertido. Ocio pasivo se asoció a introversión y no tuvo relación con la EFS.

Palabras clave: estrategias pro-felicidad, escala CEA-EAP(HIS).
\end{abstract}

\begin{abstract}
The scale of happiness increase (HIS, of Tkach and Lyubomirsky, 2006) to measure behavioural strategies of increased happiness, was adapted to the Chilean context with the name of questionnaire of strategies to increase positive moods (CEA-EAP), with 44 items in 4-point likert format. This, together with the EFS (Lyubomirsky y Lepper 1999) was answered by 443 undergraduates (global Cronbach's alpha =.80), who self-rated as extra or

Introverted. The ANAFAC showed an 8 factors structure -mostly with good reliability- that differed from the original. The strategies of mental and self-care control or positive re-evaluation and positive reorientation of attention, the recreational socialization or celebrate and, to a lesser extent but also other three different strategies were associated with happiness (EFS), and to the self-rating of Extrovert. Passive leisure was associated with introversion and had no relationship with the EFS.

Keywords: pro-happiness strategies, CEA-EAP (HIS) scale.
\end{abstract}

\footnotetext{
Correspondencia: Emilio Moyano. Email: emoyano@utalca.cl.
}

Agradecimientos: al Proyecto de investigación Fondecyt n 1131152 en cuyo último año se dio término y fue enviado a publicación el presente artículo. 


\section{Introducción}

Ha sido sugerido que el comportamiento que despliegan las personas puede resultar predictor de hasta un $40 \%$ de los niveles de felicidad de las mismas (Lyubomirsky, Sheldon y Schkade, 2005) y, dado que aquel es susceptible de ser modificable, resulta útil y relevante identificar cuáles de entre ellos son más productores o predictores de felicidad. Los estudios han estado enfocados en los posibles mecanismos comportamentales -incluidos aspectos cognitivos y emocionales-involucrados en la felicidad, así como en las estrategias que los individuos utilizan para alcanzarla (Lent, Singley, Sheu, Gainor, Brenner, Treistman y Ades, 2005).

Las personas utilizan mecanismos de autorregulación para controlar sus estados de ánimo pero la revisión de la literatura respecto de cómo el comportamiento influye en la felicidad muestra que esto ha sido poco investigado y aún menos se ha estudiado como medir aquel. Un trabajo señero ha sido el de Tkach y Lyubomirsky (2006), quienes crearon un cuestionario (HIS, "Estrategias para aumentar la felicidad") para medir las conductas que utilizan las personas para aumentar su felicidad. Este está conformado por 8 dimensiones (Tabla 1): "Afiliación social”; "Fiestas y clubes" (vinculada a actividades de sociabilización, incluyendo consumo de drogas legales e ilegales); "Control mental y Auto-cuidado", (vinculada a la reorientación de la atención y pensamiento positivo); "Persecución de objetivos instrumentales" (vinculada a la modificación de la situación y logro); "Ocio pasivo" (integrando actividades como comer, dormir y ver TV); "Ocio activo" (vinculada al ejercicio y actividades artísticas), la religión e intentos directos.

La evidencia confirma que los comportamientos y actividades medidas en el HIS se asocian a felicidad. Primero, las conductas de Afiliación y Festejar (o de socialización) son una primera fuente de felicidad: el apoyo social suele aumentar los estados de ánimos positivos (Lyubomirsky, Sheldon y Schkade, 2005; Schnittker, 2008). También, Diener y Seligman (2002) reportaron que las personas más felices dedicaban gran cantidad de tiempo para relacionarse con amigos, cultivando relaciones constructivas. Estudiantes universitarios que tienen numerosos amigos en la red social Facebook presentan un ajuste social positivo (Kalpidou, Costin y Morris, 2011). Con respecto a género, se ha señalado que las mujeres utilizan con mayor frecuencia que los hombres el apoyo social para disminuir los estados de ánimo negativos (Melin, Fugl-Meyer y Fugl-Meyer 2003; Thayer, Newman y McClain, 1994).
La evidencia también confirma que las actividades de Control mental y Auto-cuidado, vinculadas a la reorientación de la atención, al pensamiento positivo y los pensamientos y conductas asociados, son otra fuente de felicidad. Dirigir deliberadamente la atención sobre los pensamientos o actividades que se realizan en el momento presente (Erisman y Roemer 2010), evitar prestar atención a las situaciones negativas de la vida, enfocarse en las circunstancias positivas, o concentrarse en las cosas que podrían ser aún mejor, se asocian a la felicidad. En sentido similar y vinculado al comportamiento emocional, comunicarse y celebrar eventos positivos con los demás -o capitalización social- también está asociado a la felicidad (Langston, 1994). En relación a los estilos de pensamiento se ha encontrado que frente a situaciones estresantes las personas más optimistas tienden a utilizar estrategias de afrontamiento adaptativas, buscando más apoyo social, siendo más persistentes y efectivas en la resolución de problemas, lo que las lleva a ser más felices que las personas que tienen pensamientos pesimistas (Scheier, Weintraub y Carver, 1986). Por otra parte, niveles más altos de optimismo se relacionan con una mayor felicidad en los momentos de adversidad. Así, el optimismo ha sido relacionado con mayores niveles de compromiso para hacer frente a los obstáculos y reducir las estrategias evasivas de afrontamiento. El pensamiento optimista está asociado con la adopción de medidas proactivas para proteger la salud, mientras que el pensamiento pesimista se asocia con conductas perjudiciales para aquella. El optimismo ha sido relacionado con un esfuerzo mayor para satisfacer aspiraciones académicas y de mejores ingresos y, aparentemente, los optimistas tienen mejores relaciones sociales que los pesimistas (Carver, Scheier y Segerstrom, 2010), puntuando alto en estabilidad emocional y en responsabilidad (Pedrosa, Celis-Atenas, Suárez-Álvarez, García-Cueto, Muñiz, 2015).

Los estudios también muestran que perseguir objetivos instrumentales o las actividades instrumentales y de modificación de la situación para obtener metas o lograr objetivos, son otra fuente de emociones positivas. Existe evidencia de que la consecución de objetivos personales en el trabajo se asocia con aumento del bienestar subjetivo (Sheldon, Kasser, Smith y Shore, 2002). Brunstein (1993) descubrió que el compromiso con los objetivos personales (la medida en que las metas personales se asocian a un fuerte sentido de determinación y, a la vez, a la voluntad de invertir esfuerzo y paciencia para llegar a la meta), la posibilidad de alcanzar efectivamente el objetivo (la percepción de las condiciones favorables y desfavorables para lograr las metas personales), y el progreso en la consecución de éstos, son 
Tabla 1. Cuestionario de Estrategias (8) y sus items-conductas para incrementar la felicidad (HIS, de Tkach y Lyubomirsky, 2006).

\begin{tabular}{|c|c|}
\hline Estrategia & Conductas \\
\hline Estrategia I. Afiliación social & $\begin{array}{l}\text { Apoyar y animar a amigos. } \\
\text { Ayudar a otros. } \\
\text { Disfrutar el momento. } \\
\text { Recibir apoyo de amigos. } \\
\text { Interactuar/comunicarse con amigos. } \\
\text { Enfocarse en mantener las relaciones. } \\
\text { Dibujar. } \\
\text { Limpiar. } \\
\text { Cultivar un sentido del humor. } \\
\text { Enfrascarse en tareas. } \\
\text { Trabajar en habilidades sociales. } \\
\text { Tratar de convertirse en una mejor persona. }\end{array}$ \\
\hline Estrategia II. Fiestas y discotecas & $\begin{array}{l}\text { Festejar. } \\
\text { Salir a un club/pub con amigos. } \\
\text { Beber alcohol. } \\
\text { Salir a conocer gente/para entretenerse. } \\
\text { Bailar. } \\
\text { Consumir drogas ilegales. } \\
\text { Quedarse en casa y disfrutar momentos tranquilos. }\end{array}$ \\
\hline Estrategia III. Control mental & $\begin{array}{l}\text { Tratar de no pensar en ser infeliz. } \\
\text { Pensar sobre qué está mal con mi vida. } \\
\text { Intentar mirar lo positivo, pero fallar. } \\
\text { Enfocarse en los aspectos negativos de la vida. } \\
\text { Tomarse la vida como es, estar contento. } \\
\text { Cultivar un punto de vista brillante. }\end{array}$ \\
\hline $\begin{array}{l}\text { Estrategia IV. Persecución de metas } \\
\text { instrumentales }\end{array}$ & $\begin{array}{l}\text { Perseguir objetivos de carrera. } \\
\text { Intentar alcanzar el potencial total. } \\
\text { Estudiar. } \\
\text { Esmerarse en cumplir las cosas. } \\
\text { Intentar de hacerlo bien en lo académico/subir las calificaciones. } \\
\text { Organizar la vida y los objetivos. }\end{array}$ \\
\hline Estrategia V. Ocio pasivo & $\begin{array}{l}\text { Ver televisión/arrendar una película. } \\
\text { Navegar en Internet. } \\
\text { Salir al cine con amigos. } \\
\text { Quedarse en casa y disfrutar de momentos tranquilos. } \\
\text { Ir de compras. } \\
\text { Dormir. } \\
\text { Leer un libro. } \\
\text { Cantar. } \\
\text { Pasar tiempo de calidad conmigo mismo. }\end{array}$ \\
\hline Estrategia VI. Ocio activo & $\begin{array}{l}\text { Intentar mantenerse saludable/en buen estado físico. } \\
\text { Ocuparme en mis hobbies. } \\
\text { Ejercitar. } \\
\text { Buscar ocupaciones que usen mis talentos. } \\
\text { Enfrascarse en tareas. } \\
\text { Bajar los niveles de estrés. } \\
\text { Ir al cine solo. }\end{array}$ \\
\hline Estrategia VII. Religión. & $\begin{array}{l}\text { Buscar apoyo en la fe. } \\
\text { Hacer actividades religiosas/rezar, etc. }\end{array}$ \\
\hline Estrategia VIII. Intentos directos & $\begin{array}{l}\text { Actuar feliz/sonreír, etc. } \\
\text { Ponerme de buen humor. } \\
\text { Decidir ser feliz. } \\
\text { Mejorar las habilidades sociales. } \\
\text { Intentar tener auto-control. } \\
\text { Escribir un diario de vida. }\end{array}$ \\
\hline
\end{tabular}


factores correlacionados positivamente con felicidad. Para ello se requiere que los objetivos sean realistas, adecuados, flexibles y concordantes con la identidad del sujeto, de lo contrario, en caso de tratarse de metas u objetivos inalcanzables, producirían consecuencias negativas (Sheldon, Ryan, Rawsthorne y Ilardi, 1997).

Por otro lado, la evidencia sobre la eficacia de las conductas de Ocio pasivo (comer, dormir y ver TV, así como a la ingesta de comida, drogas, y bebidas ilegales y legales), es más bien negativa respecto de la felicidad. Beber alcohol ha sido históricamente frecuente en la interacción social, y en los adolescentes se ha relacionado positivamente con la aceptación de los pares y un mejor ajuste psicosocial (Newcomb y Bentler, 1988; Orford, Krishnan, Balaam, Everitt y Van der Graaf, 2004). Sin embargo, también en jóvenes se ha observado una relación significativa entre tipo de apego ansioso y tendencia al consumo de alcohol como forma de afrontar estados de ánimo negativos, desencadenándose problemas con el consumo de esta sustancia (McNally, Palfai, Levine y Moore, 2003). Asimismo, consumir alcohol se ha asociado a menor rendimiento, a malestar y problemas físicos (Casswell, You Ru Quan y Huckle Taisia, 2011; Orford, Krishnan, Balaam, Everitt y Van der Graaf, 2004). Otra conducta realizada para procurarse bienestar pero que a largo plazo afecta la salud es el consumo de tabaco, que se asocia a ansiedad y menor ajuste social. El tabaquismo se asocia a menor nivel de bienestar psicológico y de satisfacción con la vida (Pahl, Brook, Koppel y Yeon, 2011). A su vez, la marihuana es una de las drogas ilegales más consumida por los jóvenes (Mcleod, Oakes, Copello, Crome, Egger, Hickman, Oppenkowski, Stokes y Davey, 2004), y está asociada a angustia, bajo autocontrol, déficit de habilidades sociales y de afrontamiento (Benda, 2005; Huebner, Shettler, Matheson, Meszaros, Piercy y Davis 2005).

Otra conducta de ocio pasivo estudiada es la ingesta de alimentos. Las personas tienden a comer para procurarse estados anímicos positivos y de felicidad (Soto y Moyano, 2010), pero eso perjudicaría su salud y su felicidad cuando se trata de ingesta compulsiva, ya que afecta la ansiedad, el estrés y los estados de ánimo (Parylak, Koob y Zorrilla, 2011). Diversas investigaciones han mostrado que las personas realizan actividades de ocio pasivo como ver TV y dormir en exceso, creyendo que estas les ayudarán a aumentar su felicidad, pero en los hechos aquellas no solo no les reportan beneficios sino que peor aún, les disminuye sus estados de ánimos positivos (Gratz, 2003). Adicionalmente, se ha observado que las conductas agresivas en la edad adulta fueron antecedidas por la exposición a contenidos televisivos violentos en la infancia (Huesmann, Moise-Titus, Podolski y Eron, 2003), y que hay una fuerte correlación entre ver televisión y síntomas depresivos durante un período de siete años entre la adolescencia y la adultez temprana (Primack, Swanier, Georgiopoulos, Land y Fine, 2009).

En la misma línea, Soto y Moyano (2010) reportan que de los comportamientos de ocio pasivo, ver TV una de las conductas más realizadas y menos saludables, junto con descansar acostándose a dormir, por parte de estudiantes universitarios y comerciantes. Estas conductas están asociadas a una deficiente salud ya que incrementan el sedentarismo, el sobrepeso y los riesgos cardiovasculares (Moyano, Icaza, Mujica, Núñez, Leiva, Vásquez y Palomo, 2011). Hamburg, McMackin, Huang, Shenouda, Widlansky, Schulz, Gokce, Ruderman, Keaney y Vita (2007) sometieron a 20 individuos sanos a 5 días de reposo en cama, ocasionándoles a corto plazo consecuencias negativas en su metabolismo; hipertensión arterial, aumento del colesterol, de triglicéridos, de insulina y una alteración de la función microvascular. En esta misma línea, Kop, Weinstein, Deuster, Whittaker y Tracy (2008) mostraron en un estudio experimental con 20 participantes impedidos de realizar actividad física durante dos semanas un aumento de síntomas de estado de ánimo negativo y de fatiga.

Conductas de ocio activo en cambio -como la práctica deportiva y de actividades artísticas y de hobbies- han resultado buenas reguladoras del estado de ánimo y de las emociones positivas. Quienes practican deporte experimentan más felicidad que quienes no, y probablemente ello se deba a la liberación de endorfinas y el efecto social de la práctica deportiva (Hsiao y Thayer, 1998; Melin, Fugl-Meyer y FuglMeyer, 2003). Los hombres tienden a realizar más ejercicio físico que las mujeres para procurarse estados de ánimo positivos (Thayer, Newman y McClain, 1994). Escuchar música, cantar, tocar instrumentos musicales, cocinar, ver programas de arte, ir a la biblioteca, leer, asistir a obras de teatro y museos, decorar la casa y pintar, han correlacionado positivamente con felicidad, independiente de la frecuencia de su práctica. Escuchar música es una de las actividades de ocio utilizada con más frecuencia y regula los estados de ánimo, provoca placer y relaja a los oyentes e incluso genera recuerdos emotivos, lo cual aumenta las emociones positivas (Laukka, 2007). A su vez, Salimpoor, Benovoy, Larcher, Dagher y Zatorre (2011) mostraron que cuando las personas escuchan canciones de su gusto su cerebro libera dopamina, neurotransmisor que refuerza la conducta de escuchar música al provocarles placer.

Finalmente, hay una relación positiva entre creencias y prácticas religiosas con felicidad, aunque de magnitud limitada y no en todos los países. La integración social, el 
apoyo instrumental y ayudar a atribuirle sentido y propósito a la vida explicarían la asociación entre conductas religiosas y felicidad (Tkach y Lyubomirsky, 2007). La religiosidad se puede vincular también a una reevaluación positiva y atribución de significado de situaciones de vida, ambas como regulación de la afectividad.

Es probable que el conjunto de prácticas de ocio referidas precedentemente para procurarse felicidad varíen transculturalmente por una parte $y$, por otra, que también sea diferente su grado de eficacia en conducir efectivamente al logro de estados de felicidad. Para verificarlo, hemos procedido a adaptar el HIS al contexto chileno. Nuestro primer objetivo es evaluar la adecuación del instrumento para medir la frecuencia de comportamientos pro-felicidad y su eficacia en conseguirla. El segundo es comprobar si estos comportamientos se asocian a un indicador de felicidad como la EFS de Lyubomirsky y Lepper (1999).

\section{Método}

\section{Participantes}

Una muestra de 443 estudiantes universitarios chilenos de la ciudad de Talca, de los cuales $61 \%$ eran mujeres y mediante un sistema on line, aceptaron un consentimiento informado y respondieron los instrumentos correspondientes. La edad media fue de 22 años con un rango de 18 a 31 años, el $61 \%$ declaró practicar alguna religión.

\section{Instrumentos}

Se utilizan dos instrumentos, la versión chilena del HIS (Tkach y Lyubomirsky, 2006) propuesta aquí con el nombre de CEA-EAP con 8 dimensiones conservadas del HIS, pero de 44 ítems y no 56 del original, al descartarse aquellos que no parecieron adecuados al contexto cultural chileno. La HIS fue traducida del inglés por un hispanoparlante nativo bilingüe al español y confrontada por la traducción obtenida de esta al inglés por un angloparlante nativo bilingüe siguiendo las normas internacionales para el efecto. El CEA-EAP de 44 ítems conservó el formato de respuesta Likert tanto para la frecuencia de cada conducta medida ( $1=$ Nunca 0 casi nunca; $2=$ A veces; $3=$ Frecuentemente; $4=$ La mayor parte del tiempo o todo el tiempo), como para la evaluación de su eficacia en aumentar la felicidad $(1=$ Nunca o casi nunca es efectiva; $2=$ Escasamente efectiva o algo efectiva; 3 = Efectiva y 4 = Bastante efectiva), pero reduciendo los 7 puntos que tiene el HIS a solo 4 puntos. Se reporta los resultados relativos a confiabilidad para la escala total y por áreas, medias y DT. El segundo instrumento administrado es la EFS de Lyubomirsky y Lepper (1999) en versión previamente adaptada a población universitaria chilena por Moyano-Díaz (2010).

\section{Descripción de las ocho sub-escalas o dimensiones del CEAP-EAP}

La sub-escala de Afiliación Social tiene 3 ítems de la HIS. Dos ítems fueron mejorados en su redacción, el primero, "Interactuar/comunicarse con amigos", fue adaptado como "Interactúo, llamo, contacto o me comunico con amigos". El segundo, "Recibo apoyo de amigos", se transformó a "Recibo o busco el apoyo de amigos" y, finalmente, se agregaron 2 ítems nuevos que para la cultura nacional parecen relevantes (Soto y Moyano, 2010): "Pololeo o busco interactuar con quien ando" ("pololeo" significa mantener una relación afectiva de cierto grado de compromiso o formalidad, y "andar" es mantener una relación afectiva sin un compromiso formal) y, por último, el ítem "Comparto con la familia".

La segunda sub-escala corresponde a la dimensión Festejar e ir a discotecas, con 6 de 7 ítems originales de la HIS, de los cuales 5 fueron conservados sin modificación. Fue modificado el ítem "Salgo a un club/pub con amigos" quedando como "Salgo a un club, o pub o bar con amigos (as)" y, por último, se eliminó el ítem "Quedarse en casa y disfrutar momentos tranquilos", ya que no parece consistente con el comportamiento a evaluar en esta dimensión.

La sub-escala de Control Mental, compuesta por 6 reactivos, 4 de los cuales pertenecen a la HIS y uno fue modificado de: "Mirar lo positivo, pero fallar" quedó como "Intento ver lo positivo que puede haber en mis errores" y el último no fue considerado: "Cultivar un punto de vista brillante", ya que su significado no resultaba claro.

La sub-escala de Persecución de metas instrumentales quedó compuesta de 3 reactivos de los 6 originales. Los ítems "Estudiar" e "Intentar hacerlo bien en lo académico/subir las calificaciones" fueron fusionados en "Estudio o busco subir mi rendimiento y alcanzar mis objetivos académicos". El ítem "Organizar la vida y los objetivos" quedó como "Organizo mi vida y mis objetivos (planifico, distribuyo mis tiempos)". Y fueron eliminados aquí 2 ítems de la HIS, el primero "Perseguir objetivos de carrera", porque alude más bien al mundo laboral, e "Intentar alcanzar el potencial total", por la ambigüedad de su significado. 
La sub-escala de Ocio Pasivo incluye 12 actividades propias del tiempo libre u ocio. De estas actividades se mantuvieron 7 ítems de la HIS, y fueron modificados los ítems "Ir de compras", "Pasar tiempo de calidad conmigo mismo" y "Dibujo" quedando respectivamente como "Voy de compras o a vitrinear", "Pasar un tiempo solo" y "Dibujo o pinto". Por último, fueron agregados dos ítems nuevos (inexistentes en la HIS), pero que han sido reportados como propios del ocio en Chile (Soto y Moyano, 2010) "Escucho Música" y "Como algo que me gusta o de mi predilección (algo rico)".

Para la sub-escala Ocio Activo fueron utilizados tres ítems y fueron fusionados otros dos: "Intentar mantenerse saludable/en buen estado físico" y "Ejercitar" en tanto se refieren a la misma actividad o propósito, quedando como; "Intento mantenerme saludable ejercitándome físicamente". Por otro lado, se adaptarón dos ítems originales de la HIS "Bajar los niveles de estres" y "Buscar ocupaciones que usen mis talentos" del modo siguiente: "Busco modos de hacer bajar o desaparecer mi estrés" y "Cultivo el ocio (actividad placentera que no busca ganancia material personal)". Finalmente no fueron incluidos del HIS los ítems "Intentar tener autocontrol" y "Enfrascarse en tareas", ya que no corresponden a lo que se espera evaluar.

La sub-escala Religión quedó conformada aquí por un solo ítem -"Busco fortalecer mi apoyo en la fe"- resultante de la fusión de dos ítems de la HIS "Busco apoyo en la fe" y "Hacer actividades religiosas/rezar", al considerarse que ambos referían a lo mismo.

La sub-escala Intentos Directos (Auto-cuidado) quedó compuesta por 3 ítems basados en los de la HIS: "Actúo Feliz y sonrío" y "Decidir ser feliz", que fueron fusionados como "Decido ser feliz, actúo feliz y sonrío". El último ítem corresponde a "Cultivo el sentido del buen humor, me pongo de buen humor", que es producto de la modificación del reactivo "Ponerme de buen humor" (tabla 2). Los ítems eliminados de la HIS lo fueron porque no tenían validez de contenido y no tenían validez cultural aparente para la cultura chilena.

\section{Procedimiento}

Se llevó a cabo un análisis factorial confirmatorio para verificar la estructura del CEA-EAP, ya que la fiabilidad de varias de las sub-escalas o dimensiones originales eran bajas (Moyano, Torres y Páez, en prensa 2016). Estas nuevas dimensiones se correlacionaron con la EFS y la autoclasificación de personalidad como extro o introvertida. Para evaluar el peso de la frecuencia y de la efectividad de las conductas pro-incremento de la felicidad, se predijo mediante una regresión múltiple la puntuación de la escala EFS a partir de aquellas (Tabla 2). Luego, fue correlacionada la frecuencia con la eficacia, y de ésta para cada sub-escala, con la EFS, con la auto-percepción de extroversión, y las variables socio-demográficas (Tabla 3 ).

\section{Resultados}

Se reporta inicialmente el resultado del análisis factorial sobre los resultados de la aplicación del CEA-EAP y algunas relaciones con los rasgos de personalidad, introversión y extroversión. Se sigue con resultados relativos a la confiabilidad de cada sub-escala, para posteriormente reportar el resultado de la capacidad del CEA-EAP para predecir la felicidad como medida por la EFS.

\section{Análisis factorial de la escala CEA-EAP o adaptación chilena de la HIS}

Para examinar la estructura de la escala CEA-EAP se llevó a cabo un análisis factorial de componentes principales con rotación varimax imponiendo 8 factores, ya que la escala original HIS era planteada con 8 factores por una parte y, por otra, porque un screen test mostró que a partir de 8 factores bajaba la varianza explicada. Los 8 factores de la CEA-EAP explican aquí el $46 \%$ de la varianza.

El primer factor explicaba el $7 \%$ de la varianza y reunía a buena parte de las conductas de control psicológico mental (tomar vida como es -o aceptación-no pensar en lo negativo y sí en lo positivo) y auto-cuidado (cultivar sentido del humor y expresar felicidad) del HIS en su versión CEA-EAP. Esta dimensión integra conductas de aceptación, de reevaluación positiva y expresión emociones positivas.

El segundo factor explicaba alrededor del $7 \%$ de la varianza y reunía las conductas de festejar o recreación, y consumo de drogas. Podemos definirla como una estrategia de socialización lúdica y de gratificación.

El tercer factor explicaba el $6.6 \%$ de la varianza y reunía las conductas de objetivos instrumentales del HIS, integrando "limpiar" que hacía parte de ocio activo en el estudio del HIS. Se puede definir como una estrategia de logro concienzudo.

El cuarto factor explicaba el $6.3 \%$ de la varianza y reunía las conductas de afiliación social del HIS, integrando también el canto, música (ocio pasivo en el HIS) y la religiosidad (que era una dimensión diferenciada en la HIS, aunque en 
Tabla 2. Matriz de componentes rotados

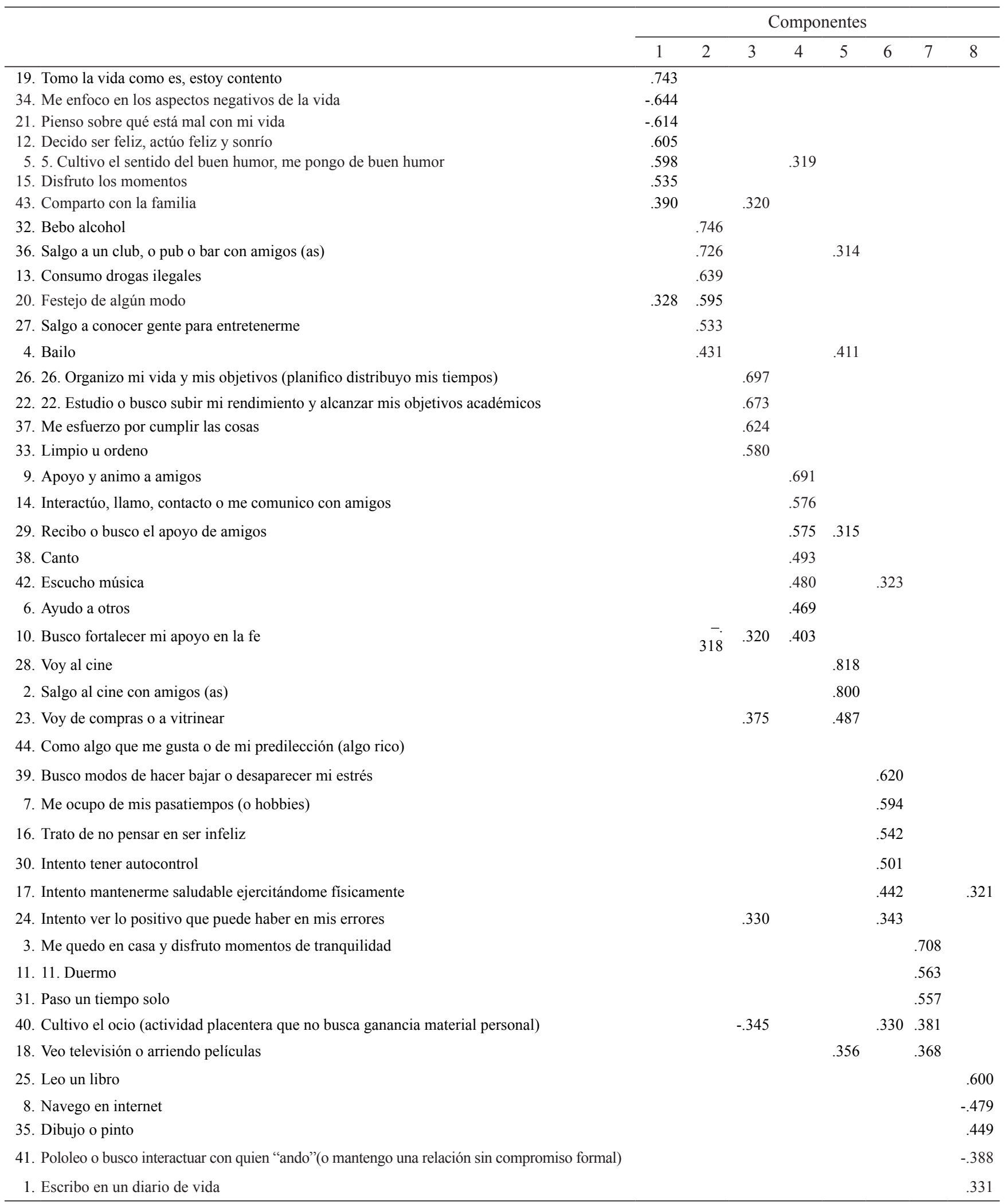

Nota. Método de extracción: Análisis de componentes principales

Método de rotación: Normalización Varimax con Kaiser.

a. La rotación ha convergido en 11 iteraciones. 
el CEA-EAP se redujo a un ítem). Es una dimensión de socialización de búsqueda y entrega de apoyo social.

El quinto factor explicaba el 5.6\% de la varianza y reunía conductas de ocio pasivo como ir al cine solo, o con amigos, y vitrinear (que se incluían en ocio pasivo en el HIS).

La sexta dimensión explicaba el 5.3\% de la varianza y reunía parte de las conductas de control psicológico como no pensar en ser infeliz, ver lo positivo de errores, auto-controlarse, con conductas de ocio activo, como hacer ejercicio, ocuparse de hobbies y buscar modos de bajar el estrés. Esta es una dimensión de manejo cognitivo, de distracción activa para disminuir el afecto negativo, de carácter probablemente más reactivo al estrés y de autocontrol activo.

El séptimo y octavo factores refieren a ocio. El primero explicaba el $4.4 \%$ de la varianza y reúne conductas de ocio pasivo solitario como quedarse en casa, dormir, ver TV o videos, estar solo y cultivar el ocio, y aislamiento social. En tanto el segundo factor explicaba el 3.9\% de la varianza y reunía a conductas de ocio pasivo como navegar por internet, leer libro, pintar, y escribir un diario de vida que podemos considerar que implican un grado de actividad intelectual mayor.

El cálculo de consistencia interna mediante coeficiente Alfa de Cronbach para cada uno de los factores precedentes muestra que el factor 1 (4 ítems) tiene .69, el 2 (6 ítems) tiene .75 , el 3 (4 ítems) tiene .68, el 4 ( 7 ítems) alcanza .67 , el 5 (3 ítems) alcanza .68, el 6 (6 ítems) alcanza .62, el 7 ( 3 ítems) alcanza .53 (eliminando el ítem ' dormir' sube a
.62), y, finalmente el factor 8 (4 ítems) obtuvo .27. Con la excepción de éste último factor, siete de los ocho factores alcanzan fiabilidades satisfactorias mostrando validez estructural de las dimensiones.

Globalmente, la suma de varianza explicada se sitúa un poco por debajo del $50 \%$ con $46 \%$. El porcentaje de varianza explicada por factor oscila entre $4 \%$ y $7 \%$ siendo bastante homogéneo inter-factores, lo que ilustra que estamos en presencia de un conjunto de factores diferenciados de importancia relativamente similar de incremento de la felicidad.

Las estrategias asociadas a la felicidad son las de control psicológico $(\mathrm{r}(443)=.63, \mathrm{p}<.001)$, el ocio activo y hacer ejercicio $(\mathrm{r}=.18, \mathrm{p}<.001)$, la búsqueda de metas instrumentales $(r=.16, p<.001)$, y en menor medida festejar $\mathrm{y}$ asistir a discotecas $(\mathrm{r}=.13, \mathrm{p}<007)$. Se asocian a la extraversión, la afiliación, el festejar y asistir a discotecas, y control psicológico auto-cuidado. Las dos estrategias de ocio pasivo se asocian a la introversión.

\section{Predicción de la felicidad a partir de las dimensiones del CEA-EAP}

Con el fin de examinar la validez predictiva de las estrategias de incremento de la felicidad se llevó a cabo una regresión múltiple de la EFS sobre aquellas. La auto-percepción de extraversión se utilizó como predictora para controlar al menos parcialmente la influencia de la personalidad. La extraversión se asocia a la felicidad y al bienestar, además

Tabla 3. Promedios, DT, terciles en base a puntuaciones totales, correlación y coeficientes beta controlando la eficacia percibida de la frecuencia de las estrategias de incremento de la felicidad

\begin{tabular}{|c|c|c|c|c|c|c|c|}
\hline \multirow[t]{2}{*}{ Dimensión } & Frecuencia & Eficacia & Alfa & Tercil Bajo & Tercil Medio & Tercil Alto & $\begin{array}{l}\text { Correlación y Beta } \\
\text { con EFS }\end{array}$ \\
\hline & M DT & M DT & & & & & \\
\hline Afiliación & 2.92 .45 & 3.25 .41 & .65 & 15 o menos & 16 a 19 & 20 o más & $\mathrm{r}=.34 * * \mathrm{~B}=.28 * *$ \\
\hline Festejar & 2.05 .47 & 2.7 .56 & .72 & 11 o menos & 12 a 18 & 19 o más & $\mathrm{r}=.29 * * \mathrm{~B}=.28 * *$ \\
\hline Control Psicológico & 2.7 .40 & 3.06 .35 & .70 & 25 o menos & 26 a 33 & 34 o más & $\mathrm{r}=.64^{* *} \mathrm{~B}=.65^{* *}$ \\
\hline Control Mental & 2.85 .48 & 3.54 .42 & .59 & & & & $\mathrm{r}=.64 \mathrm{~B}=.62$ \\
\hline Directo o Auto-cuidado & 2.47 .50 & 3.28 .54 & .48 & & & & $\mathrm{r}=.52 * * \mathrm{~B}=.46^{* *}$ \\
\hline Metas Instrumentales & 3.0 .63 & 3.07 .64 & .62 & 7 o menos & 8 a 11 & 12 o más & $\mathrm{r}=.32 * * \mathrm{~B}=.28 * *$ \\
\hline Ocio Activo & 2.37 .42 & 2.94 .49 & .48 & 12 o menos & 13 a 15 & 16 o más & $\mathrm{r}=.29 * * \mathrm{~B}=.24 * *$ \\
\hline Ocio Pasivo & 2.59 .29 & 2.93 .40 & .43 & 28 o menos & 29 a 32 & 33 o más & $\mathrm{r}=.13 * \mathrm{~B}=.03 \mathrm{~ns}$ \\
\hline
\end{tabular}

Nota. ${ }^{*} \mathrm{p}<.05, * * \mathrm{p}<.01$ 
de asociarse, como hemos visto antes, a las estrategias de actividad social.

Se llevó a cabo una regresión múltiple de las estrategias conductuales asociadas al EFS, controlando los ingresos, la posición ideológica y la extroversión, para estimar la influencia específica de estas sobre la felicidad. Las variables explicaban el $48 \%$ de la varianza $(\mathrm{F}(7,425)=57,62$, $\mathrm{p}<.001)$. En laTabla 3 se reporta promedios, desviaciones estándar y terciles en base a puntales totales, correlaciones y coeficientes beta para cada dimensión respecto de la felicidad.

Los coeficientes de un modelo de regresión en el que todas las estrategias entran simultáneamente muestran que la primera dimensión -relativa al control psicológico y auto-cuidado- es decir, de aceptación, reevaluación y expresión de emociones positivas, presenta el coeficiente multivariado más importante $(\mathrm{B}=.59, \mathrm{p}<.001)$ en tanto la distracción por ocio activo y hacer ejercicio mostraba el segundo coeficiente en importancia $(\mathrm{B}=.19, \mathrm{p}<.001)$.

La estrategia de persecución de metas o instrumental ocupa el tercer lugar en importancia $(\mathrm{B}=.16, \mathrm{p}<.001)$ $\mathrm{y}$, finalmente, el festejar e ir a discotecas o la sociabilidad recreativa mostraba el coeficiente más bajo $(\mathrm{B}=.09)$. La extraversión mostraba una influencia específica de $\mathrm{B}=.14$. En la Tabla 4 las correlaciones de cada dimensión con respecto a la EFS y la auto-clasificación de intra o extrovertido.

Para cuatro de las ocho dimensiones del CEA-EAP se observa correlaciones significativas positivas con la EFS, de las cuales la más alta es con control psicológico (autocuidado) y la más baja es festejar. A su vez, respecto de personalidad, quienes se auto-clasifican como extravertidos aparecen significativamente vinculados a festejar, a control psicológico, a afiliación o sociabilidad y a menor ocio pasivo o solitario.

\section{Discusión y conclusiones}

El CEA-EAP es un instrumento confiable y adecuado para medir la frecuencia y eficacia de los comportamientos pro-felicidad. Las estrategias de incremento de la felicidad muestran validez de criterio, ya que se asocian a la felicidad como evaluada por la EFS. Solo la religiosidad no tiene asociación con la felicidad aunque hay que señalar, por una parte, que solo fue utilizado un ítem para evaluar aquella, lo que limita la exactitud de la medida. Sin embargo, otras medidas mono-ítem también han mostrado correlaciones significativas y coherentes en este estudio. Por otra, probablemente, la paulatina secularización de la cultura católica chilena, en una muestra de estudiantes universitarios de una universidad del Estado, al menos, se refleja en la ausencia de asociación entre religiosidad y felicidad.

Se llevó a cabo un análisis factorial imponiendo ocho factores los cuales muestran coherencia y reflejan la estructura del incremento de felicidad en la cultura chilena, al menos en una muestra juvenil universitaria.

Un primer factor reunía conductas de aceptación, de reevaluación positiva y de expresión emociones positivas, similar al factor de control mental y auto-cuidado. Un segundo factor era similar al de festejar, y un tercero similar al de persecución de metas. Estos primeros dos factores eran similares a los originales de la HIS y se pueden considerar dimensiones etic de éste.

Un cuarto factor era afiliación, aunque incluía actividades artísticas, como canto, y la religión. Esto sugiere que estas actividades se realizan grupalmente en el contexto de la cultura-relativamente colectivista- chilena (Hofstede, 2001).

Un quinto factor reunía actividades de ocio pasivo, al igual que un séptimo factor que integraba dimensión de ocio pasivo y aislamiento social, y un octavo factor que reunía actividades de ocio con mayor actividad cognitiva. Aunque estas dimensiones son parcialmente de ocio pasivo, se diferenciaban en el contexto chileno respecto del resultado de la escala original de EEUU. En particular, el séptimo factor incluía una dimensión de evitación social, asociada a la introversión y no vinculada a la felicidad como los otros dos.

Un sexto factor mezclaba el control mental con ocio activo, integrando manejo cognitivo de distracción y actividades físicas de disminución disminución del afecto negativo, de carácter probablemente más reactivo al estrés y de autocontrol activo. Sus ítems mostraban más bien el intento de controlar y disminuir el afecto negativo y no tenían relación con la felicidad, probablemente porque no se relacionen con la afectividad positiva.

Los resultados muestran que el primero, segundo, tercero y sexto factores se asociaban y predecían la felicidad donde los dos primeros, el control psicológico - vinculado a la regulación por orientación de la atención, reevaluación y regulación mental y expresiva de las emociones positivas-, $\mathrm{y}$, en menor medida el festejar -asimilable a la búsqueda de apoyo social emocional y distracción-, eran estrategias eficaces para incrementar la felicidad, aun considerando el nivel de introversión-extroversión de la persona. Como sabemos, la extroversión está asociada a la afiliación, al festejar y al control psicológico, en cambio las estrategias de ocio pasivo se asocian a la introversión y no se asociaron aquí a felicidad. 
Tabla 4. Correlaciones Pearson para las 8 dimensiones del CEA-EAP con la EFS y la auto-clasificación de personalidad

Correlaciones

$\begin{array}{ll} & \text { ¿Te consideras } \\ & \text { más bien } \\ \text { Lyubomirsky t } & \text { introvertido } \\ & \text { o más bien } \\ & \text { extrovertido? }\end{array}$

\begin{tabular}{|c|c|c|c|}
\hline \multirow[t]{3}{*}{ Control auto cuidado reev } & Correlación de Pearson Sig. (bilateral) N & $.626^{* *}$ & $.230 * *$ \\
\hline & & .000 & .000 \\
\hline & & 433 & 433 \\
\hline \multirow[t]{3}{*}{ Festejar } & Correlación de Pearson Sig. (bilateral) N & $.133 * *$ & $.241 * *$ \\
\hline & & .006 & .000 \\
\hline & & 433 & 433 \\
\hline \multirow[t]{3}{*}{ Instrumental limpiar } & Correlación de Pearson Sig. (bilateral) N & $.163 * *$ & $.039 * *$ \\
\hline & & .001 & .414 \\
\hline & & 433 & 433 \\
\hline \multirow[t]{3}{*}{ Afiliación canto } & Correlación de Pearson Sig. (bilateral) N & .069 & $.209^{* *}$ \\
\hline & & .151 & .000 \\
\hline & & 433 & 433 \\
\hline \multirow[t]{3}{*}{ Ocio pasivo (cine, vitrinear) } & Correlación de Pearson Sig. (bilateral) N & .079 & .0911 \\
\hline & & .099 & .060 \\
\hline & & 433 & 433 \\
\hline \multirow[t]{3}{*}{ Distracción fisiológico } & Correlación de Pearson Sig. (bilateral) N & $.181 * *$ & -.038 \\
\hline & & .000 & .431 \\
\hline & & 433 & 433 \\
\hline \multirow[t]{3}{*}{ Ocio pasivo solo } & Correlación de Pearson Sig. (bilateral) N & -.090 & -.240 \\
\hline & & .062 & .000 \\
\hline & & 433 & 433 \\
\hline \multirow[t]{3}{*}{ Ocio pasivo cognitivo } & Correlación de Pearson Sig. (bilateral) N & .007 & $-.100 *$ \\
\hline & & .879 & 0.38 \\
\hline & & 433 & 433 \\
\hline
\end{tabular}

Nota. ** La correlación es significativa al nivel 0.01 (bilateral).

* La correlación es significativa al nivel 0.05 (bilateral). 
Los resultados confirman que las estrategias de reevaluación positiva y re-orientación positiva de la atención, las estrategias de socialización y, en menor medida, las estrategias de persecución de metas y la regulación fisiológica activa mediante ejercicio o la distracción por ocio activo, incrementan la felicidad de forma transcultural.

\section{Referencias}

Benda, B. (2005). The robustness of self- control in relation to form of delinquency. Youth y Society, 36, 418-444.

Brunstein, J. C. (1993). Personal goals and subjective well-being: A longitudinal study. Journal of Personality and Social Psychology, 65, 1061-1070

Carver, C., Scheier, M., y Segerstrom, S. (2010). Optimism. Clinical Psychology Review, 30, 879-889.

Casswell, S., Ru Quan You., y Taisia Huckle (2011) Alcohol's harm to others: reduced wellbeing and health status for those with heavy drinkers in their lives. Addiction, 106, 1087-1094.

Diener, E., y Seligman, M. (2002). Very happy people. Psychological Science, 13, 80-83.

Erisman, M., y Roemer., L. (2010). A Preliminary Investigation of the Effects of Experimentally Induced Mindfulness on Emotional Responding to Film Clips. Emotions, 10, 72-82.

Gratz, K. (2003). Risk factors and functions of deliberate self-harm: An empirical and conceptual review. Clinical Psychology: Science and Practice, 10, 192-205.

Hamburg, N., McMackin, Huang., A, Shenouda., Sh, Widlansky, M., Schulz, E., Gokce, N., Ruderman, N., Keaney, J., y Vita, J. (2007). Physical inactivity rapidly induces insulin resistance and microvascular dysfunction in healthy volunteers. Arteriosclerosis Thrombosis and Vascular Biology, 27, 2650-2656.

Hofstede, G. H. (2001). Culture's Consequences Comparing Values, Behaviors, Institutions and Organizations. Across Nations Second Edition.

Hsiao, E. T., y Thayer, R. E. (1998). Exercising for mood regulation: The importance of experience. Personality and Individual Differences, 24, 829-836.

Huebner, A., Shettler, L., Matheson, L., Meszaros, S., Piercy, P., y Davis, S. (2005). Factors associated with former smokers among female adolescents in rural Virginia. Addictive Behaviors, 30, 167-173.

Huesmann, R., Moise-Titus, J., Podolski, C., y Eron,, L. (2003). Longitudinal relations between children's exposure to TV violence and their aggressive and violent behavior in young adulthood: 1977-1992. Developmental Psychology, 39, 201-221.

Kalpidou., M., Costin., D., y Morris., J. (2011). The Relationship Between Facebook and the Well-Being of Undergraduate College Students. $C y$ berpsychology, Behavior and Social Networkin. 14, 183-189.

Kop. W., Weinstein, A., Deuster, P., Whittaker, K., y Tracy, R. (2008) Inflammatory markers and negative mood symptoms following exercise withdrawal. Brain Behvior and Imnunity, 22, 1190-1196.

Langston, C. (1994). Capitalizing on and coping with daily-Life eventsexpressive responses to positive events. Journal of Personality and Social Psychology, 67, 1112-1125.

Laukka, P. (2007). Uses of Music and Psychological Well-Being Among the Elderly. Journal of Happiness Studies, 8, 215-241.

Lent, R., Singley, D., Sheu, H., Gainor, K., Brenner, B., Treistman, D., y Ades, L. (2005). Social cognitive predictors of domain and life satisfaction: Exploring the theoretical precursors of subjective well-being. Journal of Counseling Psychology, 52, 429-442.

Lyubomirsky, S. Sheldon, K.M. y Schkade, D. (2005). Pursuing Happiness: The Architecture of Sustainable Change. Review of General Psychology, 9, 111-131.
Lyubomirsky, S., y Lepper, H. (1999). A measure of subjective happiness: Preliminary reliability and construct validation. Social Indicators Research, 46, 137-155.

Mcleod, J., Oakes, R., Copello, A., Crome, I., Egger, M., Hickman, M., Oppenkowski, T., Stokes, H., y Davey, G. (2004). Psychological and social sequelae of cannabis and other illicit drug use by young people: A systematic review of longitudinal, general population studies. The Lancet, 363, 1579-1588.

McNally, A., Palfai T., Levine, R., y Moore, B. (2003). Attachment dimensions and drinking-related problems among young adults: The mediational role of coping motives. Addictive Behaviors, 28, 1115-1127.

Melin, R, Fugl-Meyer, K., y Fugl-Meyer, A. (2003). Life satisfaction in 18-to-64 year-old Swedes: In relation to education, employment situation, health and physical activity. Journal of Rehabilitation Medicine, $35,84-90$

Moyano, E., Icaza, G., Mujica, V., Núñez, L., Leiva, E., Vásquez, M., y Palomo, I. (2011). Patrón de comportamiento tipo A, ira y enfermedades cardiovasculares (ECV) en población urbana chilena. Revista Latinoamericana de Psicología, 43, 443-453.

Moyano-Díaz, E. (2010). Exploración de algunas propiedades psicométricas de las escalas de satisfacción vital, felicidad subjetiva y autopercepción de salud. En: Moyano-Díaz, E. (Ed.) Calidad de Vida y Psicología en el Bicentenario de Chile (pp. 447-469) Marmor.

Moyano, E., Torres, M., y Páez, D. (en prensa 2016). Midiendo estrategias y eficacia para procurarse felicidad. En: Zubieta, E. et al (Ed.). Psicología Social del Bienestar en América Latina. Bs. Aires: Ed. Lugar.

Newcomb, M., y Bentler, P. (1988). Impact of adolescent drug use and social support on problems of young adults: a longitudinal study. Journal of Abnormal Psychology, 97, 64-75.

Orford, J., Krishnan, M., Balaam, M., Everitt, M., y Van der Graaf., K. (2004). University Student Drinking: the role of motivational and social factors. Drugs-Education Prevention and Policy, 11, 407-421.

Pahl, K., Brook, J., Koppel, J., y Yeon, J. (2011). Unexpected Benefits: Pathways From Smoking Restrictions in the Home to Psychological Well-Being and Distress Among Urban Black and Puerto Rican Americans Nicotine y Tobacco Research, 13, 706-713.

Parylak, S., Koob, G., y Zorrilla, E (2011). The dark side of food addiction. Physiology y Behavior, 104, 149-156.

Pedrosa, I., Celis-Atenas, K., Suárez-Álvarez, J., García-Cueto, E., y Muñiz, J. (2015). Cuestionario para la evaluación del optimismo: Fiabilidad y evidencias de validez. Terapia Psicológica, 33, 127-138.

Primack, B., Swanier, B., Georgiopoulos, A., Land, S., y Fine, M. (2009). Association Between Media Use in Adolescence and Depression in Young Adulthood. Archives of General Psychiatry, 66, 181-188.

Salimpoor, V., Benovoy, M., Larcher, K., Dagher, A., y Zatorre, R. (2011). Anatomically distinct dopamine release during anticipation and experience of peak emotion to music. Nature Neuroscience, 14, 257-355.

Scheier, M., Weintraub, J., y Carver, C. (1986). Coping with stress: Divergent strategies of optimists and pessimists. Journal of Personality and Social Psychology, 51, 1257-1264.

Schnittker, J. (2008). Happiness and Success: Genes, Families, and the Psychological Effects of Socioeconomic Position and Social Support. American Journal of Sociology 114, 233-259.

Sheldon, K., Ryan, R., Rawsthorne, L., y Ilardi, B. (1997). Trait self and true self: Cross-role variation in the Big-Five traits and its relations with psychological authenticity and subjective well-being. Journal of Personality and Social Psychology, 73, 1380-1393.

Sheldon, K., Kasser, T., Smith, K., y Shore, T. (2002). Personal goals and psychological growth: Testing an intervention to enhance goal attainment and personality integration Journal of Personality, 70, 5-31.

Soto., E., y Moyano., D. (2010). Concepto y Comportamiento de Ocio y sus Barreras en Maulinos, en el Bicentenario de la República. En: Moyano-Díaz, E. (Ed.). Calidad de Vida y Psicología en el Bicentenario de Chile (pp. 185-217).

Takch, C., y Lyubomirsky, S (2007). Spicing up kindness: The role of variety in the effects of practicing kindness on improvements in mood, happiness, and self-evaluations. Unpublished manuscript, University of California. Riverside. 
Takch, C., y Lyubomirsky,S. (2006). How do people pursue happiness? Relating personality, happiness-increasing strategies, and well-being. Journal of Happiness Studies, 7, 183-225.

Thayer, R., Newman, R., y McClain, T. (1994) Self-regulation of Mood: Strategies for Changing a Bad Mood, Raising Energy, and Reducing Tension. Journal of Personality and Social Psychology, 67, 910-925. 